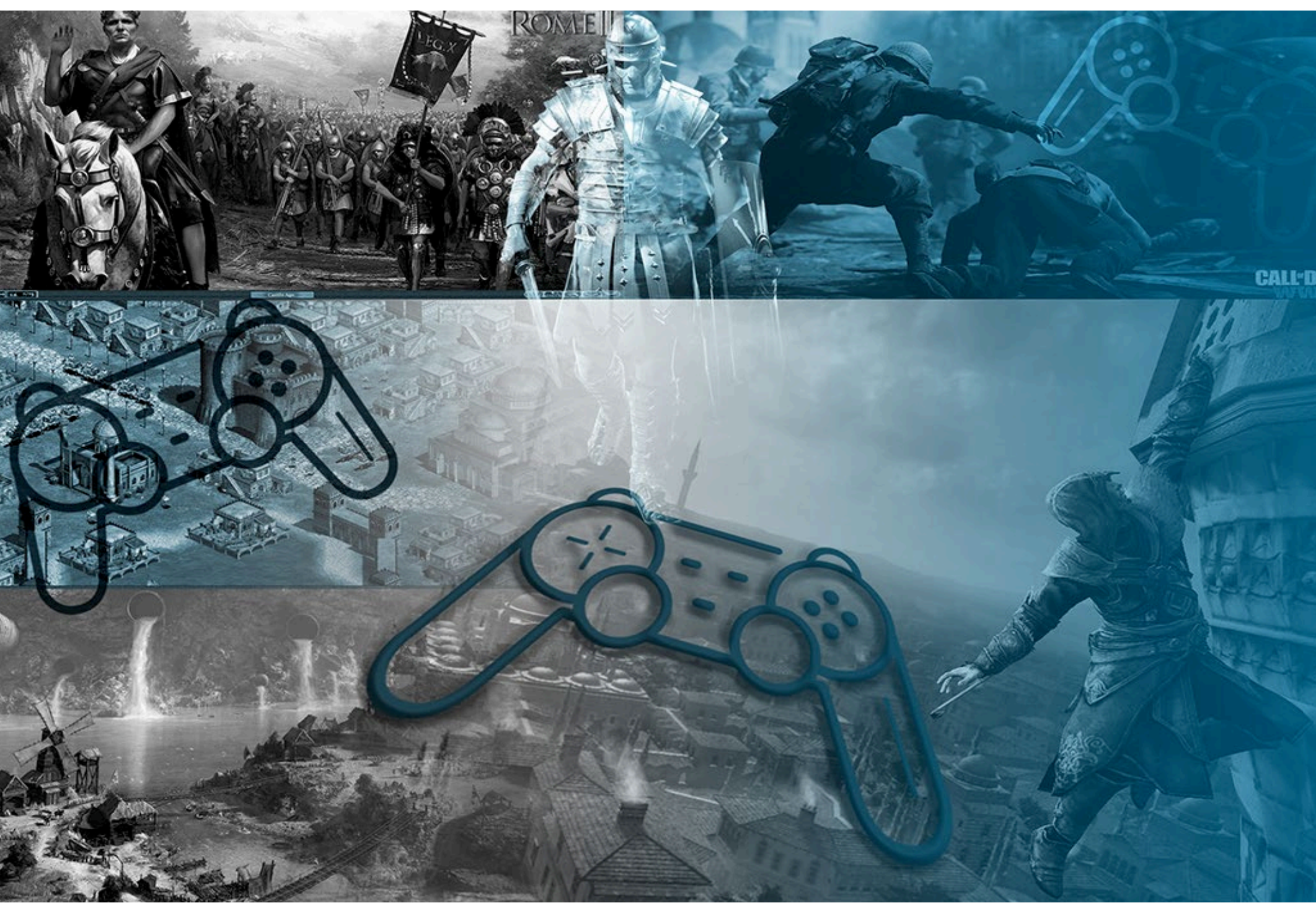

\title{
Videojuegos y enseñanza- aprendizaje de la historia. Análisis desde la producción investigativa
}

Videogames and teaching-learning of history. Analysis from the research production Jogos de vídeo e ensino-aprendizagem da história. Análise da produção de pesquisa 


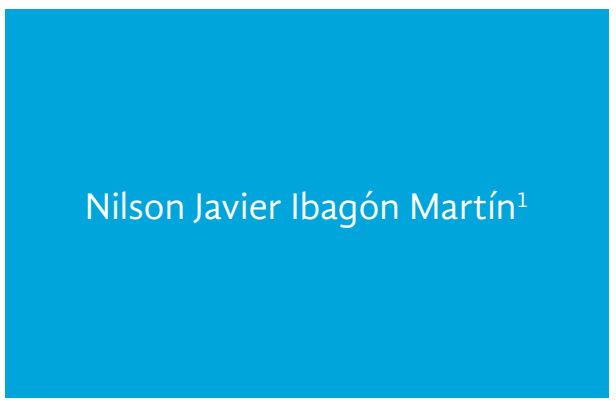

Doctorando en Educación, Universidad de Murcia. Docente Departamento de Historia, Universidad del Valle (Cali-Colombia). Integrante del Grupo de Investigaciones Históricas en Economía, Política y Educación (IHEPE); correo electrónico: nilson.ibagon@correounivalle.edu.co

Fecha de recepción: 8 de junio de 2018/ Fecha de aprobación: 26 de septiembre de 2018

\section{Resumen}

A través de la revisión hermenéutica de propuestas teóricas y prácticas, que durante las últimas dos décadas han centrado su atención en comprender la relación pedagógica entre el uso de videojuegos y la enseñanza-aprendizaje de la historia, el presente texto propone una serie de posibilidades analíticas para entender el potencial, ventajas y dificultades formativas que se derivan de este vínculo. Para ello, se definen cinco ejes de análisis que van, desde la caracterización de las experiencias, hasta el tipo de habilidades de pensamiento que se generan a través de éstas.

Palabras clave: Videojuegos, enseñanza de la historia, innovación educativa, culturas digitales.

\section{Abstract}

Through the hermeneutical review of theoretical and practical proposals, that during the last two decades have focused her attention in understanding the pedagogical relationship between the use of video games and the teaching-learning of history, this text proposes a series of analytical possibilities to understand the potential, advantages and training difficulties derived from this link. For this purpose, the article defines five axes of analysis ranging from the characterization of the experiences, up to the kind of thinking skills generated through them.

Keywords: Videogames, Teaching history, Educational innovation, Digital cultures.

\section{Resumo}

Através da revisão hermenêutica de propostas teóricas e práticas, que durante as duas últimas décadas se concentraram sua atenção em entender o relacionamento pedagógico entre o uso de videogames e o ensinoaprendizagem da história, este texto propõe uma série de possibilidades analíticas para entender o Potencial, vantagens e dificuldades de treinamento que são derivados deste link. Para isso, definir cinco eixos de análise que vão desde a caracterização das experiências, até o tipo de habilidades de pensamento que você gerar através deles.

Palavras-chave: jogos de vídeo, ensino de história, inovação culturas educacionais e digitais. 


\section{Introducción}

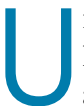
n problema central en los sistemas escolarizados actuales es la considerable distancia entre las formas de enseñar y de aprender. Esta realidad se vuelve más evidente si se tiene en cuenta que un porcentaje importante de las prácticas educativas formales es ajeno a los nuevos códigos y medios de comunicación que han sido integrados y naturalizados en la vida cotidiana de los estudiantes, en su condición de nativos digitales. Aunque las llamadas Tecnologías de la Información y la Comunicación -TIC- se han convertido en parte integral de las maneras en que las personas socializan y aprenden del mundo, en los ámbitos escolares suelen ser resistidas por algunos agentes educativos, ya sea porque les perciben como distractores y ajenas a la práctica formal del acto de educar, o porque su uso representa un reto técnico que no se está dispuesto enfrentar.

En este sentido, pese a que muchas instituciones educativas han incorporado materialmente diversas herramientas tecnológicas, su falta o simplificación de uso a la hora de desarrollar procesos de enseñanza se configura como un problema en el desarrollo de la práctica docente actual. En otras palabras, aunque discursivamente y materialmente las TIC han venido ganando una mayor presencia en la escuela, un grupo considerable de docentes sigue basando su accionar pedagógico en el método tradicional, hecho que lleva a los estudiantes a ser simples receptores sin mayor agencia (Jiménez y Cuenca, 2015).

En el caso particular de los videojuegos, artefactos culturales que durante las últimas décadas han tenido una influencia considerable en el uso del tiempo libre de niños, jóvenes y adultos -hecho que les ha llevado a convertirse en una poderosa herramienta de socialización de códigos culturales (Díez, 2009)-, han sido profunda y ampliamente subvalorados o rechazados como herramienta didáctica por buena parte de los sectores y agentes responsables de la educación formal, particularmente profesores y directivos docentes (Esnaola, 2006; Jiménez y Cuenca, 2015; Moreno, 2010; Téllez, 2015).

Sin embargo, y partiendo de la base de que no todos los videojuegos son susceptibles de un uso didáctico, desde la enseñanza de la geografía (Lotherington y Sinitskaya, 2009), las matemáticas (Goldin, 2014; Carvajal y Rojas, 2014), la ciencia (Barab, 2009; Cheng, 2015), y la educación física (Chacón, 2016), el desarrollo de investigaciones que analizan la relación entre estos artefactos culturales y la enseñanza-aprendizaje de diferentes campos disciplinares escolares ha ido en aumento.
En esta dinámica de producción, la enseñanza-aprendizaje de la historia como objeto de reflexión ocupa un lugar destacado. Un interés particular que durante las últimas dos décadas ha estado impulsado, no solo por el número de videojuegos que utilizan la historia como pieza clave para fundamentar su estructura argumentativa -principio que establece una diferencia significativa con otras disciplinas escolares-, sino asociado especialmente a la crítica establecida por diferentes sectores académicos en torno a las restricciones formativas que suponen los métodos tradicionales de enseñanza de la historia. Esta crítica ha generado una búsqueda de posibles alternativas didácticas que permitan resignificar el estudio de esta disciplina escolar, marco en el cual el análisis del uso de videojuegos se ha ido configurado como un espacio de reflexión fructífero.

A partir de ese campo específico de investigación, el presente escrito busca, desde la revisión de la literatura, analizar el potencial y limitaciones pedagógicas de dicho vínculo. Para ello, se han establecido cinco ejes de análisis: 1) La identificación de los enfoques por medio de los cuales se ha estudiado la relación; 2) Los principales avances generados o que se pueden generar en torno a habilidades cognitivas y actitudinales de los estudiantes; 3) El estudio de criterios de evaluación sobre las posibilidades educativas del uso de videojuegos en la enseñanza-aprendizaje de la historia; 4) Los riesgos y dificultades que se derivan de este uso en la práctica educativa; y 5) Los cambios que generan este tipo de innovaciones educativas en el ejercicio docente y la agencia de los estudiantes.

\section{Entre el uso de videojuegos educativos y la gamificación}

Según Van Eck (2006) existen tres vertientes de investigación sobre el uso de videojuegos en la enseñanza de la historia: 1) Innovaciones educativas en las que los estudiantes aprenden mientras crean el juego, al desempeñar el rol de diseñadores ${ }^{2}$; 2) Investigaciones que evalúan el impacto en la enseñanzaaprendizaje de la historia en videojuegos que no fueron diseñados con fines educativos; y 3) Trabajos que analizan ese impacto, pero a partir de videojuegos que fueron diseñados como estrategias educativas desde el comienzo. En dicha clasificación hay una diferencia sustancial entre la segunda y tercer vertiente, la cual se ve reflejada en los alcances formativos que se tienen en una otra.

2 Esta línea en general no tiene mucho desarrollo, en gran medida porque se requiere de un elevado grado técnico de conocimiento. 
Aunque en principio, debido a su origen, los videojuegos sujetos al estudio de la tercer vertiente y las experiencias educativas que se originan en ella deberían a priori tener los mayores impactos de aprendizaje, existen posicionamientos que ven en el uso de estos "juegos serios" menores posibilidades de innovación y motivación educativa en comparación a las experiencias educativas impulsadas por la gamificación (Kapp, 2012), esto es, procesos de enseñanzaaprendizaje que, a través del uso de videojuegos cuya naturaleza y objetivo central es el entretenimiento, logran atraer estudiantes a través de su estética, promoviendo un aprendizaje activo basado en la resolución de problemas.

La razón fundamental de esta apreciación se basa en el hecho de que la estructura narrativa y de jugabilidad -y por tanto, de motivación- de los videojuegos educativos no es equiparable a los videojuegos de entretenimiento (González, 2017; Gross, 2000). En este sentido, su radio de acción por lo general se circunscribe a la población infantil (Rubio, 2013), siendo más difícil su acogida por parte de los jóvenes. Sumado al tema generacional, su falta de dinamismo hace que estos juegos educativos tiendan a reproducir las principales deficiencias de los métodos tradicionales, lo que los lleva a sentar su base de desarrollo en la memorización de información, la descontextualización explicativa de los contenidos que abordan y la reproducción acrítica de discursos oficiales (González, 2017).

A pesar de las dificultades, hay algunas sistematizaciones de experiencias que demuestran los efectos positivos del uso de videojuegos educativos en la enseñanza de la historia. A través del análisis de resultados en aprendizajes mediados por el uso de videojuegos diseñados para enseñar y entender eventos concretos de la historia de un país, Evaristo, Navarro, Vega y Nagano (2016) demostraron comparativamente la mejora en la calidad de los resultados evaluativos de un grupo de estudiantes cuyas clases estuvieron complementadas mediante la estrategia, frente a grupos que no la tuvieron. Este éxito que poco a poco se ha venido registrando en investigaciones alrededor del mundo, se explica por dos razones fundamentales: la mediación explicativa del profesor (Evaristo, et al., 2016); y elementos asociados a las mejoras en el diseño en sí del videojuego (Egea, Ferrer y García, 2017; Šisler, Brom, Cuhra, Cinátl y Gemrot (2012), las cuales en algunos casos están más próximas a la jugabilidad que se presentan en videojuegos de entretenimiento.

Desde la perspectiva de la gamificación, estos últimos han sido estudiados principalmente a partir de dos enfoques: Su contenido intrínseco, visto desde las posibilidades educativas que se derivan de ellos, y la sistematización de experiencias prácticas basadas en su uso. Desde estos dos ejes de análisis, juegos de estrategia como "Civilization" (Chapman, 2013; Fogu, 2009; Koebel, 2017; Voorhees, 2009; Wainwright, 2014) o "Age of Empires" (Mugueta, Manzano, Alonso y Labiano, 2015) y, de ficción histórica, como “Assassin's Creed" (Rodríguez y Gutiérrez, 2016), son los más analizados y empleados.

En el caso de los estudios que se centran en el contenido, las discusiones teóricas sobre su posible uso en la enseñanzaaprendizaje de la historia en espacios escolares se enfocan esencialmente en: la estructura narrativa desde la cual se desarrollan los argumentos (Voorhees, 2009); las representaciones y demostraciones históricas (Moreno, 2010); la posibilidad de construir revisiones alrededor de argumentos históricos sobre determinados procesos y posicionamientos historiográficos de las periodizaciones de la Historia (Koebel, 2017); y la influencia de posturas ideológicas en el abordaje de las temáticas. Tales ejes de análisis, que permiten entender las concepciones de historia inmersas en las propuestas de estos artefactos tecnológicos culturales, son claves para comprender cómo el conocimiento histórico puede ser comunicado a través de estas mediaciones (Telles y Alves, 2015).

Por su parte, las investigaciones cuyo eje central es la sistematización de experiencias educativas se han concentrado en estudios dirigidos a estudiantes de secundaria (Evaristo, et al., 2016; Irigaray y Luna, 2014; McCall, 2011a; Rodríguez y Gutiérrez, 2016). Pero en los últimos años han comenzado a ser más usuales estudios que sistematizan experiencias en la educación superior. En este último campo se destacan los trabajos de: Kuran, Tozoglu y Tavernari (2018), quienes estructuraron su propuesta alrededor de actividades dirigidas a estudiantes de carreras diferentes a Historia; Wainwright (2014), cuya experiencia trabajó con estudiantes universitarios sin distinción de carreras; y Radetich y Jakubowicz (2015), quienes desarrollaron y evaluaron una estrategia dirigida a futuros profesores de Historia.

Sin embargo, independientemente del nivel educativo en el que se enfoque cada una de estas experiencias, existe un denominador común en sus conclusiones: afirmar que, pese a que la finalidad última de los videojuegos de entretenimiento de contenido histórico no es precisamente la de formar competencias y habilidades disciplinares, al ser integrados a procesos formativos escolares planificados, éstos presentan amplias posibilidades educativas en torno al desarrollo del pensamiento histórico de los estudiantes. 


\section{Desarrollo de habilidades cognitivas y actitudinales}

Desde hace por lo menos cuatro décadas, la producción investigativa en el campo de la educación histórica ha establecido la diferencia entre esquemas de trabajo afincados en ejercicios de memorización de información, y procesos formativos que buscan el desarrollo del pensamiento histórico, resaltando las ventajas de estos últimos sobre los primeros (Atkinson. 1978; Gómez y Miralles, 2017; Lee y Ashby, 1987; Barca, 2000; Wineburg, 1991; Seixas y Morton, 2013). No obstante, pese a los avances teóricos en la materia, existe una distancia considerable entre la teoría y las prácticas educativas al interior de las aulas, las cuales siguen privilegiando perspectivas afincadas en la reproducción y memorización de datos (Gómez y Miralles, 2017). Sumado a ello, el énfasis excesivo que tiene el qué sobre el cómo y el para quién en la definición de los horizontes de trabajo pedagógico, hacen que dicha distancia se amplíe.

A partir de esta problemática han surgido diferentes innovaciones educativas que buscan alternativas a la memorización de la historia. En esta búsqueda el uso de las videojuegos ha establecido posibilidades de trabajo didáctico que poco a poco han permitido explorar nuevas formas de entender la enseñanza-aprendizaje de la historia en la escuela (Gálvez, 2006). Así, aunque es alto el potencial de los videojuegos frente al desarrollo de habilidades y competencias asociadas a nuevos tipos de alfabetización necesarios en el mundo actual (Gee, 2004, 2007), la pregunta que durante la última década ha fijado el rumbo de las investigaciones sobre la relación pedagógica entre estos artefactos y la historia, entendida como saber escolar, se ha enfocado en establecer concretamente si es posible enseñar y aprender historia a través de ellos.

En el ámbito cognitivo, propuestas teóricas y prácticas han planteado que el uso de videojuegos históricos, por el hecho de facilitar la creación de narrativas propias por parte del estudiante y de promover ejercicios de contrafactualidad (Radetich y Jakubowicz, 2015) e imaginación (Arruda, 2011), amplía las posibilidades de crítica y cuestionamiento a la hora de analizar los hechos y procesos históricos.

De ahí que pensar en diferentes posibilidades de concreción del pasado facilite el desarrollo de reflexiones en torno al por qué éstas no se concretaron. En este sentido, según Ayen (2010), un trabajo sistemático y planeado en clase, que aproveche dicha posibilidad, permite ir más allá de las concepciones lineales en las cuales se apoya la enseñanza y aprendizaje tradicional de la historia, facilitando aprendizajes significativos. Por tanto, un trabajo formativo de este tipo cuestiona la idea de verdad absoluta del hecho histórico, la cual es una de las causas que impiden, en el marco de la enseñanza tradicional de la historia, generar en los estudiantes pensamiento crítico (Münzenmayer e Ibagón, 2017).

La contrafactualidad, como principio de formación que se deriva del uso de videojuegos históricos, permite así trabajar dimensiones dinámicas que otras estrategias y enfoques educativos no logran alcanzar. De esta forma, las posibilidades abiertas de construir pasados posibles en los videojuegos se contraponen a los finales unidireccionales y estáticos que, por lo general, guían las narraciones propuestas en prácticas tradicionales cuyo objetivo es la reproducción de información. En consecuencia, se potencia el trabajo pedagógico alrededor de ideas de sucesión, simultaneidad, causalidad (causa/efecto) y empatía histórica, principios básicos del pensamiento histórico.

Por ejemplo, una de las dimensiones de pensamiento histórico que más dificultades presenta al momento de enseñar historia es la de la empatía histórica. Esto es, procesos cognitivos y emocionales que permiten al estudiante analizar y entender un pasado distante descentrándose de los valores que marcan su presente. Sin embargo, indagaciones que han identificado las ventajas del uso de videojuegos en la mejora de la educación histórica de niños y jóvenes escolarizados, han registrado importantes avances en este tema específico (Chapman, 2013; Kuran, et al., 2018; Jiménez y Cuenca, 2015; Radetich y Jakubowicz, 2015; Rejack, 2007). Desde estas perspectivas, los videojuegos con contenidos históricos generan una inmersión activa del estudiante, al representar formas de ser y estar de un determinado contexto temporal, permitiéndole reconocer los rasgos específicos de una realidad ajena a su cotidianidad por medio de la vivencia -inmersión visual y auditiva ligada a la caracterización de la arquitectura, vestimenta, espacios naturales, etc.-.

Es importante en este punto tener presente que el objetivo del uso de videojuegos debe estar centrado en la posibilidad de desarrollar y adquirir ideas previas que faciliten a los estudiantes el tránsito hacia el análisis de conceptos de mayor complejidad, teniendo en cuenta el grado de escolaridad en el que se encuentran. Así, por ejemplo, temas como la contingencia en la historia (Kuran, et al., 2018), la multicausalidad (Cuenca y Martín, 2010; Valverde, 2008), el cambio histórico (Koebel, 2017), la crítica historiográfica (Wainwright, 2014), van siendo asimilados gradualmente e integrados al aparato conceptual explicativo de los estudiantes.

De forma complementaria a estos avances cognitivos, las investigaciones destacan el desarrollo de capacidades axiológicas, actitudinales y sociales que viabilizan y fortalecen los procesos de aprendizaje, las cuales, según Pindado (2005), son transversales. Desde estas dimensiones, aspectos relacionados con la motivación 
y la interacción de los estudiantes, producidas por el dinamismo que define la simulación histórica propuesta por los videojuegos, son principios que se destacan al marcar una diferencia sustancial con estrategias convencionales como lo puede ser la clase magistral (Ayén, 2010; Irigaray y Luna, 2014; McCall, 2011a; Koebel 2017; Téllez, 2015; Wainwright, 2014).

De ahí que la apatía de los estudiantes -factor actitudinal que se configura como uno de los problemas más comunes en los procesos de enseñanza escolar de la historia- sea de cierta forma superada (Rodríguez y Gutiérrez, 2016), impulsando y fortaleciendo, tanto ejercicios de aprendizaje colaborativo en los cuales estudiantes con mayor solvencia en el juego apoyan a los que presentan algún tipo de dificultad (Gros y Garrido, 2008), como prácticas vinculadas a secuencias didácticas que, al estar relacionadas directamente con la resolución de problemas, desarrollan un aprendizaje motivacional colaborativo y activo que impacta positivamente a los estudiantes en su proceso de aprendizaje (Cuenca y Martín, 2010; Cuenca, Martín y Estepa, 2011; Irigaray y Luna, 2014; Jiménez y Cuenca, 2015).

\section{Algunos criterios de evaluación del alcance del uso de los videojuegos en el aprendizaje de la historia}

Aunque el campo específico de diseño de indicadores concretos de evaluación del impacto de los videojuegos en los procesos de aprendizaje escolar es escaso, durante los últimos años se han comenzado a gestar iniciativas investigativas en este sentido (Apperlay y Beavis, 2013; Beckert, 2011; Madeiros y Schimiguel, 2012), las cuales han buscado identificar con mayor precisión si un determinado videojuego o experiencia educativa basada en ellos puede potencialmente permitir alcanzar determinados objetivos educacionales.

A partir de esta lógica, en el campo específico de los videojuegos de carácter histórico, se han venido constituyendo propuestas de evaluación que se enfocan, por una parte, en los efectos de su uso y, por otra, en la pertinencia de su contenido como discurso a ser utilizado para enseñar. Así, por ejemplo, desde la primera perspectiva, McCall (2011b) establece cinco principios para evaluar una estrategia educativa innovadora que integre los videojuegos como dinamizadores de la enseñanza-aprendizaje de la historia: a) Cuestionar las imprecisiones del videojuego; b) Reconocer el potencial de las simulaciones para el estudio del pasado; c) Generar procesos de distinción entre las narrativas historiográficas y la simulación históricamente válida propuesta por el videojuego; d) Facilidad y tiempo para desarrollar el proceso; y e) El conocimiento del profesor del juego y su sistema.
Por su parte, Egea, Ferrer y García (2017) establecieron un modelo de evaluación a partir del análisis de impacto del uso de un videojuego diseñado para aprender el valor del patrimonio histórico, el cual estaba dirigido específicamente a estudiantes de secundaria. Por medio del uso de pre test y post test determinaron la cantidad y calidad de contenidos históricos que los estudiantes lograron incorporar a sus explicaciones luego de utilizar el videojuego.

Ya en el campo específico de análisis de contenido, Kee (2011), a través de la formulación de dos preguntas, propone el establecimiento de posibles principios de evaluación de videojuegos históricos: 1) ¿Cuál es la concepción de historia que tiene?; 2) ¿Cómo es narrado y representado un conjunto de eventos? A través de modelos como los anteriormente descritos es posible establecer con mayor claridad, no solo el potencial, sino las posibles dificultades y problemas en la planeación y desarrollo de experiencias innovadoras que recurran a este tipo de mediaciones.

\section{Problemas y dificultades}

Algunas experiencias de carácter práctico en aula han demostrado que, pese a las ventajas y elementos positivos formativos que se derivan del uso de videojuegos en las clases de historia, existen algunos riesgos derivados, ya sea de la implementación como tal de la estrategia, del esquema narrativo en sí de algunos videojuegos, o de la disposición de los estudiantes hacia el desarrollo de actividades complementarias.

En el primer caso, las dificultades que representa asegurar condiciones óptimas para desarrollar las estrategias didácticas -equipos, espacios, número de estudiantes- (Radetich y Jakubowicz, 2015) y la falta de claridad en los objetivos que se persiguen (Gálvez, 2006), se convierten en los factores más comunes de riesgo. En el segundo caso, la difusión de imágenes distorsionadas de la historia ${ }^{3}$ (Clyde, Hopkins y Wilkinson, 2012; Fogu, 2009; Jiménez y Cuenca, 2015; Robinson, 2012; Schut, 2007; Téllez e Iturriaga, 2014) o simplificadas (Kusiak, 2002), y la falta de discusión del estudiantado frente al enfoque eurocéntrico desde el cual se ancla la narrativa de la mayoría de juegos comerciales con trasfondo histórico (Irigaray y Luna, 2014; Kuran, et al., 2018), se constituyen en las dificultades más registradas.

En un tercer caso, las variables problémicas se centran en la sobre-estimulación de los estudiantes en el aula (Mugueta, et al.,

\footnotetext{
Para algunos autores esta variable no sería considerada un problema, en tanto asumen que toda representación del pasado finalmente está determinada por una serie de selecciones intencionadas y por lo tanto de reducciones (Chapman, 2012).
} 
2015), que les llevaría a poner en un tercer plano de prioridad al aprendizaje; y en la falta de disposición de algunos de ellos hacia el desarrollo de actividades complementarias al uso de videojuegos, como puede ser la realización de lecturas y consultas externas orientadas a fortalecer las discusiones de clase (Wainwright, 2014). En otras palabras, pueden existir estudiantes que aprueben la dimensión de entretención de la estrategia y no la de aprendizaje.

\section{Transformaciones en las agencias} de profesores y estudiantes

Sin embargo, más allá de las dificultades asociadas en buena medida al accionar de maestros y alumnos, diferentes investigaciones han identificado que el uso de videojuegos en clase de historia impacta y transforma sus roles tradicionales en el desarrollo del proceso de enseñanza-aprendizaje de contenidos de carácter histórico. En tal sentido, se ha podido establecer que, a través de este tipo de experiencias, los maestros pueden pasar, de ser transmisores de información, a ser facilitadores y guías (Jiménez y Cuenca, 2015; MacCall, 2011a; Téllez, 2015; Watson, Mong y Harris, 2011), y los estudiantes pueden dejar su posición pasiva como simples receptores de datos, para asumir un rol activo y crítico que les permite desarrollar creativamente niveles de inferencia distintos a los que comúnmente conocemos (Radetich y Jakubowicz, 2015; Téllez, 2015).

De esta forma, prácticas educativas desarrolladas desde lógicas unidireccionales en las que el docente impone los ritmos de trabajo escolar y los discursos a incorporar -mediados por lo general a través de clases magistrales-, comienzan a dar paso a prácticas que reconocen, tanto los saberes previos de los estudiantes (Ayen, 2010), como su capacidad para construir conocimiento. A partir de esta resignificación de roles se modifica el sentido y metodología de la evaluación, un proceso central del acto educativo (Kuran, et al., 2018). Lo que prima no es la acumulación de información descontextualizada, sino la posibilidad de sentar posicionamientos críticos.

A partir de este horizonte de trabajo, los horizontes de indagación más comunes han sido: la evaluación de la percepción de los participantes, culminada la experiencia (Evaristo, et al., 2016; Radetich y Jakubowicz, 2015); la ejecución de tareas académicas concretas a lo largo de las clases (Irigaray y Luna, 2014); el establecimiento de escenarios de discusión al interior de los salones de clase (Wainwright, 2014); y la triangulación de información obtenida por estos instrumentos (Rodríguez y Gutiérrez, 2016).

No obstante, las transformaciones de las agencias de profesores y estudiantes en el proceso de enseñanza-aprendizaje de la historia a través del uso de videojuegos, dependen en gran medida de la claridad en los objetivos que estructuran la presencia de estos últimos en las dinámicas de clase. Por esta razón, las estrategias didácticas les emplean no pueden estar sujetas al azar y la improvisación. Por el contrario, implican el desarrollo de ejercicios de planificación sustentados en saberes propios del área de conocimiento de la historia y experticias alrededor de las TICs.

En este sentido, es indispensable complementar esta estrategia didáctica con actividades asociadas al desarrollo del pensamiento histórico. Las investigaciones prácticas que se han desarrollado sobre el uso de videojuegos en las clases de historia insisten, desde ese horizonte de trabajo, en la necesidad de desarrollar ejercicios educativos simultáneos, vinculados, por ejemplo: al trabajo con fuentes históricas (Irigaray y Luna, 2014); la búsqueda de información complementaria (Gálvez, 2006); y el desarrollo de clases magistrales y la producción de ensayos por parte de los estudiantes (Kuran, et al., 2018). La conjugación de estas mediaciones con el uso de videojuegos es la que asegura que los resultados de aprendizaje tiendan a ser más precisos y controlables, lo cual, en últimas, implica una mayor validez curricular.

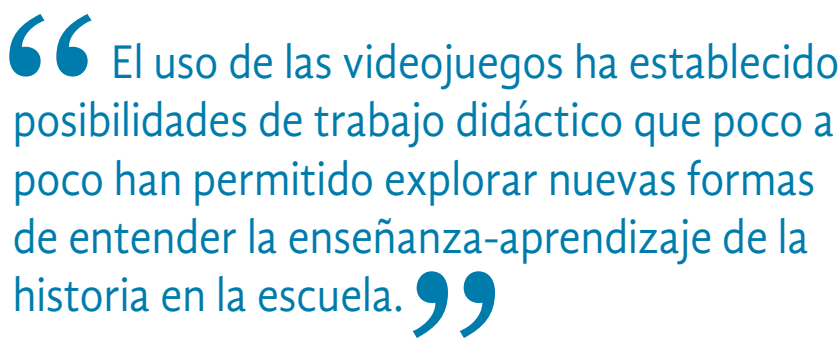

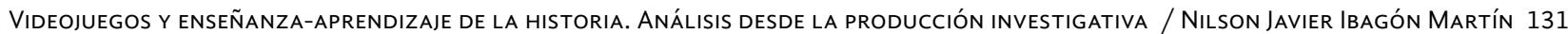




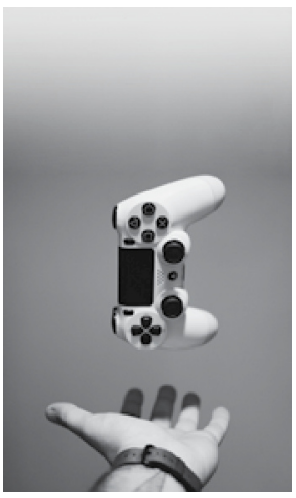

\section{Conclusiones}

El campo de reflexión investigativa, acerca del uso de los videojuegos como herramientas didácticas que pueden mejorar los procesos de enseñanza-aprendizaje escolares, se ha fortalecido durante las últimas dos décadas. En este proceso de posicionamiento como campo específico de investigación educativa, la enseñanzaaprendizaje de la historia ha tenido un lugar preponderante como objeto de estudio. No obstante, y pese a que desde dimensiones teórico-prácticas se han demostrado los efectos positivos de usar videojuegos en las clases de historia y ciencias sociales, en aspectos cognitivos y actitudinales de los estudiantes y en la estructura del quehacer docente de los profesores, aún sigue siendo extraña, ajena y resistida en muchos espacios escolares la posibilidad de desarrollar innovaciones de este carácter.

Esta realidad es de especial preocupación, no por la falta de uso de los videojuegos en sí, los cuales solo se constituyen como una posibilidad dentro de cientos de lenguajes tecnológicos que se pueden emplear para potenciar los aprendizajes históricos, sino por la incoherencia que genera no integrar las TICs y nuevos lenguajes digitales a procesos formativos relacionados con la enseñanzaaprendizaje de la historia que, discursivamente -en muchos casos desde el currículo oficial- estructuran su razón de ser a partir del desarrollo del pensamiento crítico de los estudiantes.

En este punto es clave entender que la integración de la cultura digital en los procesos educativos no se asegura mediante su presencia física en sí, ni en su uso per se, sino en el sentido que define su vínculo con los procesos formativos que se buscan llevar a cabo mediante su intermediación. De ahí que el reto de integrar efectivamente lenguajes y artefactos tecnológicos -como el que pueden constituir los videojuegos- a la enseñanza escolar de la historia, más allá de los avances significativos que se han tenido durante los últimos años en el campo de la investigación, siga teniendo vigencia y urgencia.

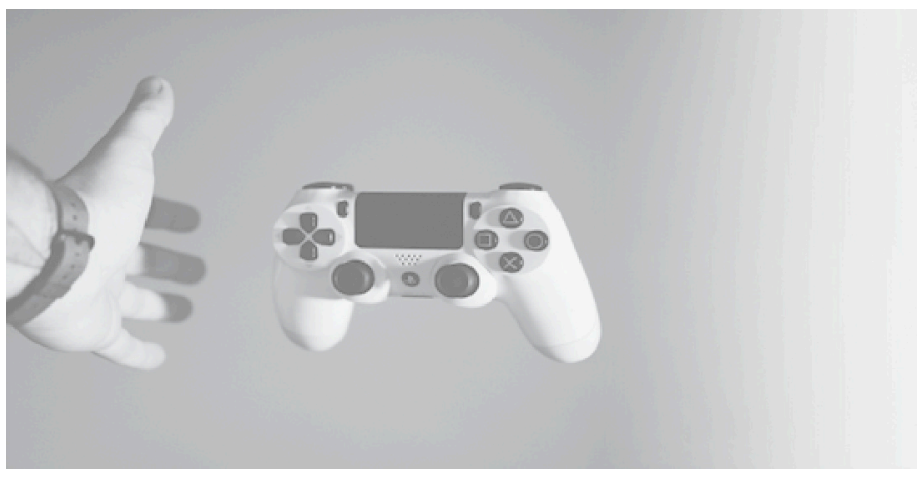




\section{Referencias}

Apperley, T., y Beavis, C. (2013). A model for critical games literacy. E-learning \& Digital Media, Vol. 10, No. 1 , pp. 1-12.

Arruda, E. (2011). O papel dos videogames na aprendizagem de conceitos e analogias históricas pelos jovens. Ensino Em Re-Vista, No. 2, pp. 287-297.

Atkinson, R. (1978). Knowledge and explanation in history. Londres: MacMillan.

Ayen, F. (2010). Aprender historia con el juego "Age of Empires". Clio, No. 36. Obtenido desde http://clio. rediris.es/n36/otros/Aprender_historia_con_el_juego_Age_of_Empires.pdf

Barab, S. (2009). Transformational play as a curricular scaffold: Using Videogames to support Science Education. Journal of Science Education and Technology, Vol. 18, No. 4, pp. 305-320.

Barca, I. (2000). O pensamento histórico dos jovens. Braga: Universidade do Minho.

Beckert, K. (2011). The Magic Bullet: A tool for Assessing and Evaluating Learning Potential in Games. International Journal of Game-Based Learning, Vol. 1, No. 1, pp. 19-31.

Carvajal, G., y Rojas, P. (2014). El videojuego como agente motivador en el aprendizaje de las matemáticas. Grafías disciplinares de la UCP, No. 27, pp. 45-56.

Chacón, R. (2016). Videojuegos activos como recurso TIC en el aula de educación física: estudio a partir de parámetros de ocio digital. Digital Education Review, No. 29, pp. 112-123.

Chapman, A. (2012). Privileging form over content: Analysing historical Videogames. Journal of Digital Humanities. Obtenido desde http://journalofdigitalhumanities.org/1-2/privileging-form-overcontent-by-adam-chapman/

Chapman, A. (2013). Is Sid Meir's Civilization history? Rethinking History, Vol. 17, No. 3, pp. 312-332.

Cheng, M. (2015). The use of serious games in science education: a review of selected empirical research from 2002 to 2013. Journal of Computers in Education, Vol. 2, No. 3, pp. 353-375.

Clyde, J., Hopkins, H., y Wilkinson, G. (2012). Beyond the "historical" simulation: theories of history to inform scholarly game design. Loading, Vol. 6, No. 9, pp. 3-16.

Cuenca, J., y Martín, M. (2010). La resolución de problemas en la enseñanza de las ciencias sociales a través de videojuegos. Revista Iber, No. 63, pp. 32-42.

Cuenca, J., Martín, M., y Estepa, J. (2011). Historia y videojuegos: una propuesta de trabajo para el aula de $1^{\circ}$ de ESO. Revista Iber, No. 69, pp. 64-73.

Díez, E. (2009). Sexismo y violencia: La socialización a través de los videojuegos. Feminismo/s, No. 14, pp. 35-52. 
TEMA: INDUSTRIAS CULTURALES Y EDUCACIÓN

Egea, A., Ferrer, L., y García A. (2017). Videojuegos, historia y patrimonio: primeros resultados de una investigación educativa en educación secundaria. Revista interuniversitaria de investigación en Tecnología Educativa, No. 2, pp. 28-40.

Esnaola, G. (2006). Claves culturales en la construcción del conocimiento ¿Qué enseñan los videojuegos? Buenos Aires: Alfagrama Ediciones.

Evaristo, I., Navarro, R., Vega, V., y Nakano, T. (2016). Uso de un videojuego educativo como herramienta para aprender historia del Perú. Revista Iberoamericana de Educación a Distancia, Vol. 19, No. 2, pp. 35-52.

Fogu, C. (2009). Digitalizing historical consciousness. History and Theory, No. 47, pp. 103-121.

Gálvez, M. (2006). Aplicaciones de los videojuegos de contenido histórico en el aula. Revista ICONO14, No. 4, pp. $217-230$.

Gee, J. (2004). Lo que nos enseñan los videojuegos sobre el aprendizaje y el alfabetismo. Archidona: Aljibe.

Gee, J. (2007). What video games have to teach us about learning and literacy. New York: Palgrave Macmillan.

Goldin, A. (2014). Far transfer to language and math of a short software-based gaming intervention. PNAS, Vol. 111, No. 17, pp. 6443-6448.

Gómez, C., y Miralles, P. (2017). Los espejos de Clío. Usos y abusos de la historia en el ámbito escolar. Madrid: Silex.

González, M. (2017). Los videojuegos del Bicentenario de la Independencia de México. Usos y abusos de la identidad, el héroe y la historia. Antropología Experimental, No. 17, pp. 305-324.

Gros, B. (2000). La dimensión socioeducativa de los videojuegos, Edutec, No. 12, pp. 1-11.

Gros, B., y Garrido, J. (2008). Con el dedo en la pantalla: El uso de un videojuego de estrategia en la medición de aprendizajes curriculares. Teoría de la educación: Educación y Cultura en la Sociedad de la Información, Vol. 9, No. 3, pp. 108-129.

Irigaray, M., y Luna, M. (2014). La enseñanza de la historia a través de videojuegos de estrategia. Dos experiencias áulicas en la escuela secundaria. Clío \& Asociados, No. 18-19, pp. 411-437.

Jiménez, R., y Cuenca, J. (2015). El uso didáctico de los videojuegos. Concepciones e ideas de futuros docentes de ciencias sociales. Clio. History and history teaching, No. 41. Obtenido desde http://clio. rediris.es/n41/articulos/JimenezCuenca2015.pdf.

Kapp, K. (2012). The Gamification of Learning and Instruction: Game-Based Methods and Strategies for Training and Education. San Francisco: John Wiley \& Sons.

Kee, K. (2011). Computerized History Games: Narrative Options. Simulation \& Gaming, No. 42, pp. 423-440.

Koebel, G. (2017). Simulating the Ages of Man: periodization in Civilization V and Europa Universalis IV. Loading, Vol. 10, No. 17, pp. 60-76. 
Kuran, M., Tozoglu, A., y Tavernari, C. (2018). History-Themed Games in History Education: Experiences on a Blended World History Course. Obtenido desde https:// arxiv.org/abs/1805.00463

Kusiak, J. (2002). Virtual Historiography: How history is presented en entertainment. Based Computer Games. Kirksville: Truman State University.

Lee, P., y Ashby, R. (1987). Discussing the evidence. Teaching History, No. 47, pp. 11-14.

Lotherington, H., y Sinitskaya, N. (2009). Gaming geography: educational games and literacy development in the grade 4 classroom. Canadian Journal of Learning and Technology, Vol. 35, No. 3, pp. 1-19.

McCall, J. (2011a). Gaming the Past: Using Video Games to teach secondary History. New York: Routledge.

McCall, J. (2011b). On Evaluating Simulation Games for Classroom Use. Obtenido desde http:// teachinghistory.org/nhec-blog/25225

Madeiros, M., y Schimiguel, J. (2012). Uma abordagem para avaliação de jogos educativos: ênfase no ensino fundamental. Revista Novas Tecnologias na Educação, No. 10, pp. 1-10.

Moreno, M. (2010). Aprender historia en ambientes virtuales. Tejuelo, Vol. 9, No. 1, pp. 58-82.

Mugueta, I., Manzano, A., Alonso, P., y Labiano, L. (2015). Videojuegos para aprender Historia: una experiencia con Age of Empires. Revista Didáctica, Innovación y Multimedia. No. 32, pp. 1-13.

Münzenmayer, A., e Ibagón, N. (2017). Pensamiento crítico: ¿Competencia olvidada en la enseñanza de la historia? Entramado, Vol. 13, No. 2, pp. 186-198.

Pindado, J. (2005). Las posibilidades educativas de los videojuegos. Una revisión de los estudios más significativos. Pixel.Bit, No. 26, pp. 55-67.

Radetich, L., y Jakubowicz, E. (2015). Using Video Games for Teaching History. Experiences and Challenges. Athens Journal of History, Vol. 1, No. 1, pp. 1-14.

Rejack, B. (2007). Toward a Virtual Reenactment of History: Video Games and the Recreation of the Past. Rethinking History, Vol. 11, No. 3, pp. 411-25.

Robinson, N. (2012). Videogames, persuasion and the war on terror: escaping or embedding the militaryentertainment complex? Political Studies, Vol. 60, No. 3, pp. 504-522.

Rodríguez, M., y Gutiérrez, D. (2016). Innovación en el aula de Ciencias Sociales mediante el uso de videojuegos. Revista Iberoamericana de Educación, Vol. 72, No. 2, pp. 181-200.

Rubio, A. (2013). El pasado en tu sofá: juegos de simulación histórica en entornos computacionales portables. HER\&MUS 13, Vol. 5, No. 2, pp. 55-62.

Schut, K. (2007). Strategic simulation and our past: the bias of computer games in the presentation of History. Games and Culture, Vol. 2, No. 7, pp. 213-235. 
TEMA: INDUSTRIAS CULTURALES Y EDUCACIÓN

Seixas, P., y Morton, T. (2013). The big six historical thinking concepts. Toronto: Nelson College Indigenous.

Šisler, V., Brom, C., Cuhra, J., Cinátl, K., y Gemrot, J. (2012). Stories from the History of Czechoslovakia. A serious game for teaching History of the Czech Lands in the 20th Century. Notes on Design concepts and design process. Entertainment Computing ICEC 2012, pp. 67-74.

Telles, H., y Alves, L. (2015). Narrativa, História e Ficção: os history games como obras fronteiriças. Comunicação e Sociedade, No. 27, pp. 303-317.

Téllez, D. (2015). Plague INC.: Pandemias, videojuegos y enseñanza-aprendizaje de las Ciencias Sociales. Revista Enseñanza de las Ciencias Sociales, No. 14, pp. 135-142.

Téllez, D., e Iturriaga, D. (2014). Videojuegos y aprendizaje de la Historia: La Saga Assasin's Creed. Contextos Educativos, No. 17, pp. 145-155.

Valverde, J. (2008). Aprender a pensar históricamente con apoyo de soportes informáticos. Cultura y Educación, Vol. 20, No. 2, pp. 181-199.

Van Eck, R. (2006). Digital game-based learning: it's not just the digital natives who are restless. Educause Review, Vol. 41, No. 2, pp. 17-30.

Voorhees, G. (2009). I play therefore I am: Sid Meier's Civilization, turn-based strategy games and the Cogito. Games and Culture, Vol. 4, No. 3, pp. 254-275.

Wainwright, A. (2014). Teaching Historical Theory through Video Games. The History Teacher, Vol. 47, No. 4, pp. 579-612.

Watson, W., Mong, C., y Harris, C. (2011). A case study of the in-class use of a video game for teaching high school history. Computers \& Education, No. 56, pp. 466-474.

Wineburg, S. (1991). Historical problem solving: a study the cognitive processes used in the evaluation of documentary and pictorial evidence. Journal of Educational Psychology, Vol. 83, No. 1, pp. 73-87. 